\section{ScIrP with ZrNiAl-type Structure}

Ulrike Pfannenschmidt, Ute Ch. Rodewald, and Rainer Pöttgen

Institut für Anorganische und Analytische Chemie, Universität Münster, Corrensstraße 30, 48149 Münster, Germany

Reprint requests to R. Pöttgen.

E-mail: pottgen@uni-muenster.de

Z. Naturforsch. 2011, 66b, 205-208;

received December 1, 2010

The phosphide ScIrP was synthesized from the elements in a bismuth flux and characterized by powder and singlecrystal X-ray diffraction: $\mathrm{ZrNiAl}$ type, $P \overline{6} 2 m, Z=3, a=$ $637.2(3), c=389.2(2) \mathrm{pm}, w R 2=0.0280,250 F^{2}$ values, 15 variables. The two crystallographically independent phosphorus sites have tricapped trigonal-prismatic metal coordination $\mathrm{P} 1 \mathrm{Ir}_{3} \mathrm{Sc}_{6}$ and $\mathrm{P} 2 \mathrm{Ir}_{6} \mathrm{Sc}_{3}$. The shortest interatomic distances occur for Ir-P (244-251 pm) within the 3D [IrP] network in which the scandium atoms fill cavities of coordination number $15(4 \mathrm{Sc}+6 \mathrm{Ir}+5 \mathrm{P})$.

Key words: Phosphide, Scandium, Crystal Structure

\section{Introduction}

Ternary equiatomic phosphides $T T^{\prime} \mathrm{P}$ of the electron-poor $(T)$ and electron-rich $\left(T^{\prime}\right)$ transition metals have intensively been studied in the least 40 years with respect to their crystal structures [1-9] and physical properties [10-12]. Depending on the size and the electron count of the transition metal, these phosphides either crystallize with the orthorhombic TiNiSi [13] or the hexagonal ZrNiAl-type structure [14-16]. Very interesting magnetic properties have been reported for a series of compounds with chromium and manganese as transition metal components. Such phosphides and arsenides show magnetic ordering within the chromium or manganese substructure with the highest Curie temperature of $496 \mathrm{~K}$ for MnRuAs [12]. Another topic concerns superconductivity. The highest transition temperature of $13.3 \mathrm{~K}$ has been observed for $\mathrm{ZrRuP}[10,11]$.

While many of such phosphides have been synthesized with the group IV and group V transition metals, with scandium so far only hexagonal $\mathrm{ScCoP}[5]$ and orthorhombic ScNiP [9] have been reported. During our recent flux-assisted synthesis of rare earth metal-
Table 1. Crystal data and structure refinement for ScIrP, space group $P \overline{6} 2 m, Z=3$.

\begin{tabular}{|c|c|}
\hline Refined composition & ScIrP \\
\hline Formula weight, $\mathrm{g} \mathrm{mol}^{-1}$ & 268.13 \\
\hline Unit cell dimensions, pm & $a=637.2(3)$ \\
\hline (Guinier data) & $c=389.2(2)$ \\
\hline Cell volume, $\mathrm{nm}^{3}$ & 0.1369 \\
\hline Calculated density, $\mathrm{g} \mathrm{cm}^{-3}$ & 9.76 \\
\hline Crystal size, $\mu \mathrm{m}^{3}$ & $30 \times 30 \times 40$ \\
\hline Transm. ratio (max/min) & 1.86 \\
\hline Absorption coefficient, $\mathrm{mm}^{-1}$ & 76.9 \\
\hline Detector distance, $\mathrm{mm}$ & 60 \\
\hline Exposure time, $\min$ & 6 \\
\hline$\omega$ range; increment, deg & $0-180 ; 1.0$ \\
\hline Integr. param. $\mathrm{A}, \mathrm{B}, \mathrm{EMS}$ & $13.2 ; 3.2 ; 0.012$ \\
\hline$F(000), \mathrm{e}$ & 339 \\
\hline$\theta$ range for data collection, deg & $3.6-34.8$ \\
\hline Range in $h k l$ & $\pm 10, \pm 10, \pm 6$ \\
\hline Total no. reflections & 2189 \\
\hline Independent reflections / $R_{\text {int }}$ & $250 / 0.0511$ \\
\hline Reflections with $I \geq 2 \sigma(I) / R_{\sigma}$ & $246 / 0.0237$ \\
\hline Data / ref. parameters & $250 / 15$ \\
\hline Goodness-of-fit on $F^{2}$ & 1.215 \\
\hline$R 1 / w R 2$ for $I \geq 2 \sigma(I)$ & $0.0135 / 0.0278$ \\
\hline$R 1 / w R 2$ for all data & $0.0142 / 0.0280$ \\
\hline Flack parameter $x$ & $-0.01(2)$ \\
\hline Extinction coefficient & $0.0181(12)$ \\
\hline Largest diff. peak / hole, e $\AA^{-3}$ & $1.49 /-1.76$ \\
\hline
\end{tabular}

iridium-phosphides $[17,18$, and refs. therein], we obtained ScIrP. The synthesis and structure of this phosphide are reported herein.

\section{Experimental Section}

Synthesis

ScIrP was obtained from a bismuth flux [19]. Starting materials were pieces of scandium (Smart Elements, $99.999 \%$ ), iridium powder (Heraeus, $>99.9 \%$ ), red phosphorus (Hoechst, Knapsack, ultrapure), and bismuth shots (ABCR $\mathrm{GmbH},>99.99 \%)$. The elemental components in a molar ratio of $1: 1: 1: 30(\mathrm{Sc}: \mathrm{Ir}: \mathrm{P}: \mathrm{Bi})$ were sealed in an evacuated silica tube. The ampoule was positioned in a muffle furnace, heated to $770 \mathrm{~K}$ at a rate of $50 \mathrm{~K} \mathrm{~h}^{-1}$ and kept at that temperature for $24 \mathrm{~h}$. Subsequently the temperature was raised to $1370 \mathrm{~K}$ at the same rate, and the sample was annealed at that temperature for $100 \mathrm{~h}$ followed by slow cooling to $970 \mathrm{~K}$ at a rate of $2 \mathrm{~K} \mathrm{~h}^{-1}$ and further to $570 \mathrm{~K}$ at a rate of $4 \mathrm{~K} \mathrm{~h}^{-1}$. The bismuth flux was slowly dissolved by a 1:1 molar mixture of $\mathrm{H}_{2} \mathrm{O}_{2}$ (ACROS, $35 \%$ ) and glacial acetic acid (VWR International). The resulting sample was washed with demineralized water containing monoclinic $\mathrm{IrP}_{2}$ as the main component and aggregates of pillar-shaped ScIrP crystals with metallic luster as reaction products. ScIrP can 
Table 2. Atomic coordinates and anisotropic displacement parameters $\left(\mathrm{pm}^{2}\right)$ for ScIrP. $U_{\text {eq }}$ is defined as one third of the trace of the orthogonalized $U_{\mathrm{ij}}$ tensor. The anisotropic displacement factor exponent takes the form: $-2 \pi^{2}\left[\left(h a^{*}\right) 2 U_{11}+\ldots+\right.$ $\left.2 h k a^{*} b^{*} U_{12}\right] . U_{23}=U_{13}=0$.

\begin{tabular}{lllllllll}
\hline Atom & Wyck. site & $x$ & $y$ & $z$ & $U_{11}$ & $U_{22}$ & $U_{33}$ & $U_{12}$ \\
\hline Sc & $3 g$ & $0.5773(3)$ & 0 & $1 / 2$ & $65(5)$ & $52(6)$ & $56(6)$ & $26(3)$ \\
Ir & $3 f$ & $0.24809(5)$ & 0 & 0 & $33(1)$ & $38(1)$ & $98(1)$ & $19(1)$ \\
P1 & $2 c$ & $2 / 3$ & $1 / 3$ & 0 & $46(6)$ & $U_{11}$ & $66(11)$ & $23(3)$ \\
P2 & $1 b$ & 0 & 0 & $1 / 2$ & $92(9)$ & $U_{11}$ & $80(16)$ & $46(5)$ \\
\hline
\end{tabular}

also be obtained via flux-free synthesis from the elements, however, byproducts like $\operatorname{IrP}_{2}$ and other yet unknown phases obtained thereby could not be removed so far.

\section{EDX data}

The single crystal investigated on the diffractometer was studied by EDX using a Zeiss EVO MA10 scanning electron microscope with $\mathrm{Sc}$, Ir and $\mathrm{GaP}$ as standards for the semiquantitative measurements. The analyses indicated Sc, Ir and $\mathrm{P}$ as the main components. Due to the significant overlap of the phosphorus $K(2.1013 \mathrm{keV})$ and iridium $M(1.977 \mathrm{keV})$ lines, a quantitative analysis was not possible. Other impurity elements (especially bismuth incorporation from the flux) were not observed.

\section{X-Ray diffraction}

The polycrystalline ScIrP sample was characterized by $\mathrm{X}$-ray powder diffraction on a Guinier camera (equipped with a Fujifilm image plate system, BAS-1800) using $\mathrm{Cu} K_{\alpha 1}$ radiation and $\alpha$-quartz $(a=491.30, c=540.46 \mathrm{pm})$ as an internal standard. The hexagonal lattice parameters were deduced from a least-squares refinement of the powder data. To ensure correct indexing, the experimental pattern was compared to a calculated one [20] using the positional parameters obtained from the structure refinement.

Pillar-shaped crystal fragments of ScIrP were separated from the flux-grown sample by mechanical fragmentation. The crystals were glued to quartz fibers using beeswax and were characterized by Laue photographs on a Buerger camera (white molybdenum radiation, image plate technique, $\mathrm{Fu}$ jifilm, BAS-1800) in order to check their suitability for an intensity data collection. The data set was collected at room temperature by use of an IPDS II diffractometer (graphitemonochromatized $\mathrm{Mo} K_{\alpha}$ radiation; oscillation mode). A numerical absorption correction was applied to the data set. All relevant crystallographic data and details of the data collection and evaluation are listed in Table 1.

\section{Structure refinement}

The structural relation of ScIrP with the ZrNiAl-type structure [14-16], space group $P \overline{6} 2 m$, was already evident from the X-ray powder diffraction data. Consequently we

\begin{tabular}{llll}
\hline Sc: & 1 & P2 & $269.4(2)$ \\
& 4 & P1 & $272.3(1)$ \\
& 2 & Ir & $286.1(2)$ \\
& 4 & Ir & $304.7(2)$ \\
& 4 & Sc & $329.8(2)$ \\
Ir: & 2 & P1 & $244.1(1)$ \\
& 2 & P2 & $250.7(1)$ \\
& 2 & Ir & $273.8(1)$ \\
& 2 & Sc & $286.1(2)$ \\
& 4 & Sc & $304.7(2)$ \\
P1: & 3 & Ir & $244.1(1)$ \\
& 6 & Sc & $272.3(1)$ \\
P2: & 6 & Ir & $250.7(1)$ \\
& 3 & Sc & $269.4(2)$ \\
\hline
\end{tabular}

Table 3. Interatomic distances (pm) in ScIrP. All distances within the first coordination spheres are listed.

used the structural parameters of isotypic ScAgSn [21] as starting values, and the structure was refined with anisotropic displacement parameters for all atoms with SHELXL-97 (full-matrix least-squares on $F_{\mathrm{o}}{ }^{2}$ ) [22]. All sites were fully occupied within two standard deviations. Refinement of the correct absolute structure was ensured through a calculation of the Flack parameter [23, 24]. The refinement smoothly converged to the residuals listed in Table 1 . The atomic parameters and interatomic distances are listed in Tables 2 and 3 .

Further details of the crystal structure investigation may be obtained from Fachinformationszentrum Karlsruhe, 76344 Eggenstein-Leopoldshafen, Germany (fax: +49-7247808-666; e-mail: crysdata@ fiz-karlsruhe.de, http://www.fizinformationsdienste.de/en/DB/icsd/depot_anforderung.html) on quoting the deposition number CSD-422403.

\section{Discussion}

ScIrP crystallizes with the ZrNiAl [14-16] type structure. A projection of the structure is shown in Fig. 1. Striking structural motifs are two types of tricapped trigonal prisms around the phosphorus atoms,

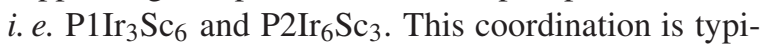
cal for metal-rich phosphides. The shortest interatomic distances in the ScIrP structure occur for Ir-P (244$251 \mathrm{pm}$ ), slightly longer than the sum of the covalent radii for $\mathrm{Ir}+\mathrm{P}$ of $236 \mathrm{pm}$ [23]. Within the threedimensional [IrP] network (Fig. 1) we observe also short $\mathrm{Ir}-\mathrm{Ir}$ bonds (the triangles of the $\mathrm{P} \mathrm{Ir}_{3} \mathrm{Sc}_{6}$ prisms) 


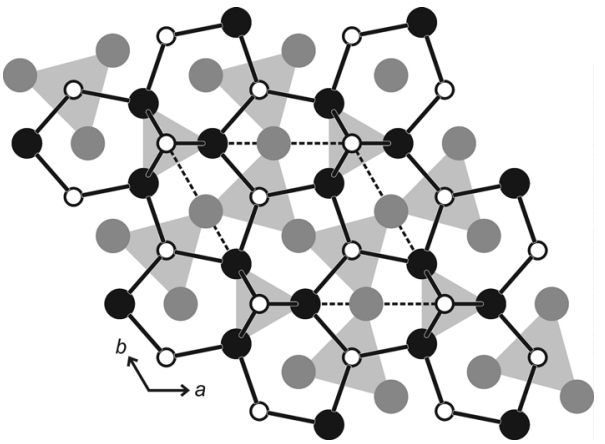

Fig. 1. Projection of the ScIrP structure onto the crystallographic $x y$ plane. Scandium, iridium, and phosphorus atoms are drawn as medium-grey, black, and open circles, respectively. The three-dimensional [IrP] network and the trigonalprismatic phosphorus coordination (light-grey shading) are emphasized.

of $274 \mathrm{pm}$, similar to those in $f c c$ iridium (272 pm) [24]. As expected for a metal-rich phosphide, various $\mathrm{Sc}-\mathrm{Sc}$ interactions also occur in the ScIrP structure. The shortest Sc-Sc distance of $330 \mathrm{pm}$ compares well with those in the structure of $h c p$ scandium $(6 \times 325$ and $6 \times 331 \mathrm{pm}$ ) [24]. For further crystal chemical details on the broad family of $\mathrm{ZrNiAl}$ intermetallics we refer to review articles [25,26, and refs. therein].

Finally we need to comment on the $U_{33}$ displacement parameter of the iridium atoms, which is about three times higher than $U_{11}$. Such a displacement might point to superstructure formation, similar to ScAgSn [21] or ScPtSn [27]. We have carefully checked the reciprocal space with respect to weak superstructure reflections which would enlarge the unit cell. No additional reflections were detected. Magnetic susceptibility measurements revealed Pauli paramagnetism and gave no hint for superconductivity down to $2.5 \mathrm{~K}$.

\section{Acknowledgements}

This work was financially supported by the Deutsche Forschungsgemeinschaft. We thank Dipl.-Chem. M. Eul for the susceptibility measurement.
[1] P. Villars, L. D. Calvert, Pearson's Handbook of Crystallographic Data for Intermetallic Phases, $2^{\text {nd }}$ ed., American Society for Metals, Materials Park, 1991; desk edition 1997.

[2] S. Rundqvist, P.C. Nawapong, Acta Chem. Scand. 1966, 20, 2250.

[3] R. Guérin, M. Sergent, Mater. Res. Bull. 1977, 12, 381.

[4] N. Chaichit, P. Chalugune, S. Rukvichai, P. Choosang, V. Kaewchansilp, C.-O. Pontchour, P. Phavanantha, S. Pramatus, Acta Chem. Scand. A 1978, 32, 309.

[5] W. Jeitschko, E. J. Reinbold, Z. Naturforsch. 1985, 40b, 900.

[6] G. A. Marking, H. F. Franzen, J. Alloys Compd. 1994, 204, L17.

[7] H. Kleinke, H. F. Franzen, Z. Anorg. Allg. Chem. 1996, 622, 1893.

[8] Ya.F. Lomnytska, Yu. B. Kuz'ma, J. Alloys Compd. 1998, 269, 133.

[9] H. Kleinke, H. F. Franzen, J. Solid State Chem. 1998, 137, 218.

[10] R. Müller, R. N. Shelton, J. W. Richardson, Jr., R. A. Jacobson, J. Less-Common Met. 1983, 92, 177.

[11] G. P. Meisner, H. C. Ku, Appl. Phys. A 1983, 31, 201.

[12] T. Kanomata, T. Kawashima, H. Utsugi, T. Goto, H. Hasegawa, T. Kaneko, J. Appl. Phys. 1991, 69, 4639.

[13] C. B. Shoemaker, D. P. Shoemaker, Acta Crystallogr. $\mathbf{1 9 6 5}, 18,900$.

[14] P.I. Krypyakevich, V. Ya. Markiv, E. V. Mel- nyk, Dopov. Akad. Nauk. Ukr. RSR, Ser. A 1967, 750 .

[15] A. E. Dwight, M. H. Mueller, R. A. Conner, Jr., J. W. Downey, H. Knott, Trans. Met. Soc. AIME 1968, 242, 2075.

[16] M. F. Zumdick, R.-D. Hoffmann, R. Pöttgen, Z. Naturforsch. 1999, $54 b, 45$.

[17] U. Pfannenschmidt, U.Ch. Rodewald, R. Pöttgen, Z. Anorg. Allg. Chem. 2010, 636, 314.

[18] U. Pfannenschmidt, U.Ch. Rodewald, R. Pöttgen, Z. Naturforsch. 2011, 66b, 7.

[19] M. G. Kanatzidis, R. Pöttgen, W. Jeitschko, Angew. Chem. 2005, 117, 7156; Angew. Chem. Int. Ed. 2005, 44, 6996.

[20] K. Yvon, W. Jeitschko, E. Parthé, J. Appl. Crystallogr. 1977, 10, 73.

[21] C.P. Sebastian, L. Zhang, C. Fehse, R.-D. Hoffmann, H. Eckert, R. Pöttgen, Inorg. Chem. 2007, 46, 771.

[22] G. M. Sheldrick, SHELXL-97, Program for the Refinement of Crystal Structures, University of Göttingen, Göttingen (Germany) 1997. See also: G. M. Sheldrick, Acta Crystallogr. 2008, A64, 112.

[23] J. Emsley, The Elements, Oxford University Press, Oxford 1999.

[24] J. Donohue, The Structures of the Elements, Wiley, New York, 1974.

[25] E. Parthé, L. Gelato, B. Chabot, M. Penzo, K. Cenzual, R. Gladyshevskii, TYPIX - Standardized data and crystal chemical characterization of inorganic 
structure types, Gmelin Handbook of Inorganic and Organometallic Chemistry, $8^{\text {th }}$ ed., Springer, Berlin, 1993.

[26] M. F. Zumdick, R. Pöttgen, Z. Kristallogr. 1999, 214, 90.
[27] R. Mishra, R. Pöttgen, R.-D. Hoffmann, H. Trill, B. D. Mosel, H. Piotrowski, M. F. Zumdick, Z. Naturforsch. 2001, 56b, 589. 